\title{
Review of: "Down syndrome is an oxidative phosphorylation disorder"
}

\author{
Jacqueline London $^{1}$ \\ 1 Paris Diderot University
}

Potential competing interests: The author(s) declared that no potential competing interests exist.

The paper by Pilar Bayoan-Bafaluy et als from Carmen Martinez-Cué team is an interesting new approach of molecular aspects of Down syndrome. Down syndrome (DS) is a multigene multisytem disorder which affects young, adults and old adults persons present most of them in the general population stream as their life expectancy is around 70 years nowdays in developped countries.

The complete or part of a third copy of chromosome 21 (HSA21) is the cause of DS, but even if all the genes ot this small chromosome are known at the genomic level, their functions and their interactions are still poorly understoof for most of them.

The approach by these authors give new and very promissing light of a general molecular mechanism involved in all the aspects of the syndrome and especially those affected by bioenergetics activities in the patients. The authors describe the OXPHOS system which provides the energetics activities of the neurons both during devolpment and aging.

The OXPHOS system is related to both the electron transport chain and the ATP synthase and it is known that oxidative phosphorylation dysfunctions in fibroblasts, lymphocytes, platelets from individuals with DS are present. The OXPHOS deficit system is not a side effect but an early and general event in individuals with DS and therefore the authors suggest that Down syndrome can be considered as an OXPHOS disorder. Thus, they examine the HSA21 genes responsible for the OXPHOS deficits. Among them, the $A P P$ and its derived peptides (AICD and CT99) can interact with OXPHOS, the cystathionine b synthase (CBS )which is an inhibitor of the respiratory CIV, the liver type phosphofructose kinase (PFK) which could play a key role in the metabolic balance regulation between glycolysis and OXPHOS functions. Three other genes which have been rather largely investigated could be also involved : Dyrk1A which might have an indirect effect on mitochondrial biogenesis and OXPHOS function through modulation of two other genes ; the regulator calcineurin gene (RCAN1) and it overexpression reduces mitochondrial mass and oxygen consumption both in human neuroblatoma cells and in b murine cells; the $B A C H 1$ gene negatively and indirectly regulates mitochondrial biogenesis.

In conclusion, the authors investigate some drugs which might be beneficial, either by inhibiting HSA21 genes such as PFK, DYRKIA, CBS, through their reduction of OXPHOS functions, or by acting on other gens or pathways and modulating OXPHOS -related genes 
In conclusion, this paper shed a new light to investigate either on IPS human DS cells or murine and cell lines models, the roles of the OXPHOS functions in Down syndrome in the perspective of the amelioration of the quality of life of patients with DS 